\title{
INJECTIVE MODULES UNDER FLAT BASE CHANGE
}

\section{HANS-BJ $\varnothing R N$ FOXBY}

ABSTRACT. It is proved that an injective module after a flat change of base is of pointwise finite injective dimension if and only if all the fibers at points associated to the injective module are Gorenstein rings (or trivial).

Notation. Throughout this note $f: A \rightarrow B$ will be an homomorphism of commutative noetherian rings $A$ and $B$ (with identities; $f(1)=1$ ) making $B$ into a flat $A$-module.

For a prime ideal $p$ in $A$ (respectively a prime ideal $q$ in $B$ ) we will write $k(p)=(A / p) \otimes_{A} A_{k} \quad$ (respectively $\left.l(q)=(B / q) \otimes_{B} B_{q}\right)$. Let $F(p)$ denote the fiber of $f$ at $p$; that is $F(p)=k(p) \otimes_{A} B\left(=B_{p} / p B_{p}\right.$ where $\left.B_{p}=B \otimes_{A} A_{p}\right)$. The prime ideals in $F(p)$ are of the form $q^{\prime}=q F(p)$ where $q$ is a prime ideal in $B$ lying over $p$, and we will write $C(q)=F(p) q^{\prime}=k(p) \otimes_{A} B_{q}$.

Finally $\operatorname{id}_{B} M=$ the injective dimension of the $B$-module $M\left(\mathrm{id}_{B} 0=-1\right.$ ).

The aim of this note is to prove the following result.

Theorem 1. If $E$ is an injective A-module, then

$$
\operatorname{id}_{B}\left(E \otimes_{A} B\right)=\sup _{\text {Ass } E} \operatorname{id}_{F(\mathfrak{v})} F(p)
$$

where the supremum is taken over all prime ideals $\not$ in $A$ associated to $E$.

Remark. The proof of the above result uses the main idea in the following theorem, which is Proposition 9.6 of Hartshorne [5, p. 297] (and the first paragraph of its proof). We include this result here to provide examples of flat base changes with nice fibers, and thereby applications of Theorem 1 .

Theorem 2 (Hartshorne). If $A$ is a Gorenstein ring, then the following three statements are equivalent:

(i) $B$ is a Gorenste in ring.

(ii) For all $\$$ which are maximal among the contracted prime ideals in $A$ the fiber $F(p)$ is a Gorenstein ring.

Received by the editors April 22, 1974.

AMS (MOS) subject classifications (1970). Primary 13C10, 13D05; Secondary $13 \mathrm{H} 10$. 
(iii) For all $p$ in $\operatorname{Spec} A$ the fiber is either a Gorenstein ring or trivial.

A corresponding result for dualizing (or Gorenstein) complexes follows from essentially the same proof.

Now, assume that $B$ is Gorenstein while $A$ is not necessarily Gorenstein. Then for $q$ in Spec $B$ we have that $A_{\mathrm{q} \cap A}$ is Gorenstein (since $A_{\mathrm{q} \cap A} \rightarrow$ $B_{q}$ is local and thereby faithfully flat). Therefore we have the following result.

Corollary. If $B$ is a Gorenstein ring then all the fibers are Gorenstein rings or trivial.

Example 1. Let $B=A[[T]]$ and assume that $A$ is a homomorphic image of a Gorenstein ring (say $R$ ). Then all the fibers are Gorenstein rings (since the fibers of $R \rightarrow R[[T]]$ are). And $\operatorname{dim} F(p) \leq \max (1, \operatorname{dim}(A / p))$.

Example 2. Let $B=\hat{A}$, the completion of $A$ w.r.t. an ideal $\delta$. The fibers of (ii) in Theorem 2 above are of the form $A / \mathrm{m}$ where $\mathrm{m}$ is a maximal ideal containing $\delta$. Therefore if $A$ is a homomorphic image of a Gorenstein ring, then all the fibers of $A \rightarrow \hat{A}$ are Gorenstein rings or trivial. $\operatorname{dim} F(\hat{p}) \leq$

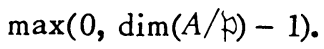

The proof of Theorem 1 starts with two easy lemmas.

Lemma 1. Let $M$ be any module over a noetherian, commutative ring $R$ and let $x$ be an integer, $x \leq \mathrm{id}_{R} M(\leq \infty)$. Then there exists a prime ideal $x$ in $R$ such that $\operatorname{Ext}_{R}^{x}(R / x, M)_{x} \neq 0$.

Proof. Let $\mathfrak{x}$ be maximal among the ideals $i$ in $R$ with $\operatorname{Ext}_{R}^{x}(R / i, M) \neq 0$. Since $R$ is noetherian there exists a chain $0=N_{0} \subset N_{1} \subset \cdots \subset N_{u-1} \subset N_{u}=$ $R / \mathfrak{x}$ of submodules of $R / \mathfrak{x}$ and prime ideals $\mathfrak{x}_{1}, \cdots, \mathfrak{x}_{u}$ in $R$ such that $N_{i} / N_{i-1} \simeq R / x_{i}$ for all $i$. Here each $x_{i}$ contains $x$ and $\operatorname{Ext}_{R}^{x}(-, M)$ is a half-exact functor, so by the maximality of $x$ we conclude that $x$ is equal to one of the $x_{i}$ 's, that is $x$ is a prime ideal. Now, if $r \in R-x$ then there is an exact sequence

$$
0=\operatorname{Ext}_{R}^{x}(R /(\mathfrak{x}+(r)), M) \rightarrow \operatorname{Ext}_{R}^{x}(R / x, M) \stackrel{r}{\longrightarrow} \operatorname{Ext}_{R}^{x}(R / x, M) .
$$

This shows that the set of zero-divisors on $\operatorname{Ext}_{R}^{x}(R / x, M)$ is contained in $x$. In particular, $\operatorname{Ext}_{R}^{x}(R / x, M)_{\mathfrak{r}} \neq 0$ as desired.

Lemma 2. Let $(R, \mathrm{~m}, k)$ and $(S, n, l)$ be noetherian, local rings and let $g: R \rightarrow S$ be a flat, local ring homomorphism. Then for an injective $R$-module E we have isomorphisms 


$$
\operatorname{Ext}_{C}^{x}\left(l, \operatorname{Hom}_{R}(k, E) \otimes_{R} S\right)=\operatorname{Ext}_{S}^{x}\left(l, E \otimes_{R} S\right) \text { for } x \geq 0
$$

where $C=S / \mathrm{mS}$.

Proof. This follows from the spectral sequence of Hartshorne [5, p. 297]. This spectral sequence was also the key in the proof of Theorem 2. Another way to prove the lemma is the following: Let $I^{*}$ be an $S$-injective resolution of $E \otimes_{R} S$. Now, since $\operatorname{Ext}_{S}^{x}\left(C, E \otimes_{R} S\right)=\operatorname{Ext}_{R}^{x}(k, E) \otimes_{R} S=0$ for $x>0$ we conclude that $\operatorname{Hom}_{S}\left(C, I^{*}\right)$ is a $C$-injective resolution of $\operatorname{Hom}_{R}\left(C, E \otimes_{R} S\right)=\operatorname{Hom}_{R}(k, E) \otimes_{R} S$, so from the identity

$$
\operatorname{Hom}_{C}\left(l, \operatorname{Hom}_{S}\left(C, I^{*}\right)\right)=\operatorname{Hom}_{S}\left(l, I^{*}\right)
$$

the result follows by passing to cohomology.

Proof of Theorem 1. Since $A$ is noetherian we can assume that $E=$ $E_{A}(A / f)$ where $k$ is a prime ideal in $A$. We are then required to show that

$$
\mathrm{id}_{B}\left(E \otimes_{A} B\right)=\mathrm{id}_{F(\mathfrak{p})} F(\mathfrak{p}) \text {. }
$$

Proof of $\leq$. Let $x \leq \mathrm{id}_{B}\left(E \mathcal{J}_{A} B\right)$ and choose, by Lemma 1, a prime ideal $q$ in $B$ such that $\operatorname{Ext}_{B}^{x}\left(B / q, E \otimes_{A} B\right)_{q} \neq 0$.

Write $S=B_{q}$ and $R=A_{q \cap A}$, so $C=S /(q \cap A) S, l=l(q)$, and $k=k(q \cap A)$. Then $\operatorname{Ext}_{S}^{x}\left(l, E \otimes_{A} S\right)=\operatorname{Ext}_{B}^{x}\left(B / q, E \otimes_{A} B\right)_{q} \neq 0$, and therefore, in particular, $\left(E \otimes_{A} R\right) \otimes_{R} S=E \otimes_{A} S \neq 0$. This shows $E_{q \cap A}=E \otimes_{A} R \neq 0$, and hence $q \cap A \geq \not$ and $E=E_{\wedge \cap A}$ is an injective $R$-module. By Lemma 2 we have

$$
\operatorname{Ext}_{C}^{x}\left(l, \operatorname{Hom}_{R}(k, E) \otimes_{R} S\right)=\operatorname{Ext}_{S}^{x}\left(l, E \otimes_{R} S\right) \neq 0
$$

and hence, in particular $\operatorname{Hom}_{A}(A / q \cap A, E) \otimes_{A} R=\operatorname{Hom}_{R}(k, E) \neq 0$; that is $q \cap A \leq p$.

Now it is proved that $p=q \cap A$, and therefore $\operatorname{Hom}_{R}(k, E) \otimes_{R} S=$ $k \otimes_{R} S=C$. The isomorphism (*) shows $\operatorname{Ext}_{C}^{x}(l, C) \neq 0$; that is $\mathrm{id}_{C} C \geq x$. Since $C=C(q)$ is a localization of $F(p)$ (at the prime ideal $q F(p)$ ) we have $\mathrm{id}_{F(p)} F(p) \geq x$ as desired.

Proof of $\geq$. Since $F(p)$ is a noetherian ring, $\mathrm{id}_{F(p)} F(p)=\operatorname{sup~id}_{C(q)} C(q)$ where the supremum is taken over all prime ideals $q$ in $B$ lying over $p$.

So let $q$ be such a prime ideal and let $x$ be any integer such that $\operatorname{dim} C(q) \leq x \leq \mathrm{id}_{C(} C(q)$. Then, by Lemma 2 and Bass [1], we have

$$
\operatorname{Ext}_{B_{q}}^{x}\left(l(q), E \otimes_{A} B_{q}\right)=\operatorname{Ext}_{C(q)}^{x}(l(q), C(q)) \neq 0
$$

and therefore $\operatorname{id}_{B}\left(E \otimes_{A} B\right) \geq \mathrm{id}_{B_{q}}\left(E \otimes_{A} B_{q}\right) \geq x$.

Example 3. Assume that $A$ is a homomorphic image of a Gorenstein 
ring, and let $E$ be an injective $A$-module. Then it follows from Examples 1 and 2 that we have

and

$$
\operatorname{id}_{A}[[T]]\left(E \otimes_{A} A[[T]]\right) \leq \max \left(1, \operatorname{dim}_{A} E\right),
$$

$$
\operatorname{id}_{\hat{A}}\left(E \otimes_{A} \hat{A}\right) \leq \max \left(0, \operatorname{dim}_{A} E-1\right) .
$$

Here, $\operatorname{dim}_{A} E$ is the dimension of $\operatorname{Supp}_{A} E$ in $\operatorname{Spec} A$, so $\operatorname{dim}_{A} E=$ $\sup _{\text {Ass } E} \operatorname{dim}(A / p)$.

Example 4. Two other standard examples of homomorphisms $A \rightarrow B$ with nice fibers are the following:

(a) If $A \rightarrow B$ is formally smooth (cf. e.g. Matsumura [4, Chapter 28, $\mathrm{G}$ and $\mathrm{K}]$ ) then the fibers are regular rings (or trivial).

(b) If $A$ is local and $A^{b}$ denotes its henselization then the fibers of $A \rightarrow A^{b}$ are products of fields (cf. e.g. Raynaud [7, p. 95]).

As a corollary of Theorem 1 we obtain a slightly stronger result dealing with an injective $R$-module $E$ under a flat change of base $R \rightarrow S$ where the commutative rings $R$ and $S$ are not necessarily noetherian. But $E$ is required to be $\Sigma$-injective, that is, any direct sum of copies of $E$ is injective, cf. e.g. Beck [2]. Now let $\beta$ denote the set of prime ideals in $R$ contained in an associated prime ideal of $E$. Then $R$ is $\beta$-noetherian in the sense of Claborn and Fossum [3] (cf. also [2]) that is, to each ideal $i$ in $R$ there exists a subideal $j$ which is finitely generated such that $j R_{p}=i R_{p}$ for all $\mathfrak{h}$ in $\Re$. Put $\mathfrak{\Omega}=\{q \in \operatorname{Spec} S \mid q \cap R \in \mathfrak{B}\}$.

Corollary. Let $g: R \rightarrow S$ be a flat ring homomorphism and let $E$ be a $\Sigma$-injective $R$-module. Assume that $S$ is $\Omega$-noetherian where $\cong$ is the set of prime ideals in $S$ lying over a prime ideal which is contained in an ideal from Ass $_{R} E$. Then

$$
\operatorname{id}_{S}\left(E \otimes_{R} S\right)=\sup _{\text {Ass } E} \operatorname{id}_{F(p)} F(p)
$$

where $F(\eta)$ is the fiber of $g$ at $p$.

Furthermore, $E \otimes_{R} S$ has an injective resolution by $\Sigma$-injective $S$ modules.

Proof. First assume that $E$ is indecomposable, and hence $E=E_{R}(R / \hbar)$ (cf. [2]). In this case $\mathfrak{D}=\{q \mid q \cap R \leq \mathfrak{p}\}$, so $B=S_{p}$ is noetherian. Since each injective $B$-module is a $\Sigma$-injective $S$-module, it is clear that $E \otimes_{R} S=$ $E_{p} \otimes_{R} S=E \otimes_{R} B$ has an injective resolution by $\Sigma$-injective $S$-modules. $A=R_{p}$ is a noetherian ring, $E=E_{A}(A / p A)$, and $F(\not)$ is the fiber of $A \rightarrow B$ 
at $\nmid A$. The equality follows now from Theorem 1 , since $\mathrm{id}_{B}\left(E \otimes_{A} B\right)=$ $\mathrm{id}_{S}\left(E \otimes_{R} S\right)$.

Now back to the general case, where $E$ is a direct sum of indecomposable injective modules (cf. [2]). The identity follows directly from the identity in the indecomposable case. Furthermore each of $E$ 's indecomposable components has a minimal injective resolution by $\Sigma$-injective modules, and each of these is a direct sum of modules of the form $E_{S}(S / q), q$ in $\Omega$. A direct sum of modules of this type is again $\Sigma$-injective (cf. [2]), and we are done.

Example 5 (Beck [2]). Let $E$ be a nonzero $\Sigma$-injective $R$-module. By Claborn and Fossum [3, Theorem 3.2] $R[T]$ is $\cong$-noetherian, and the fiber of $R \rightarrow R[T]$ at $k$ is $k(\not)[T]$. Therefore $E[T]$ has an injective resolution by $\Sigma$-injective $S$-modules and $\mathrm{id}_{R}[T]{ }^{E[T]}=1$.

Example 6 (Beck [2]). Let $E$ be a $\Sigma$-injective $R$-module and let $R \rightarrow S$ be a localization (that is "un épimorphisme plat" (cf. Lazard [6])). Here all ideals in $S$ are extensions of ideals in $R$, and hence $R$ is $\Omega$-noetherian and all the fibers are fields or trivial. Conclusion: $E \otimes_{R} S$ is a $\Sigma$-injective $S$-module.

Finally, I wish to thank R. M. Fossum for many helpful comments during the preparation of this manuscript.

\section{REFERENCES}

1. H. Bass, On the ubiquity of Gorenstein rings, Math. Z. 82 (1963), 8-28. MR 27 \#3669.

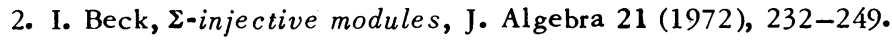

3. L. Claborn and R. Fossum, Class groups of n-noetherian rings, J. Algebra 10 (1968), 263-285. MR 39 \#5541.

4. H. Matsumura, Commutative algebra, Benjamin, New York, 1970. MR 42 \#1813.

5. R. Hartshorne, Residues and duality, Lecture Notes in Math., no. 20, Springer-Verlag, Berlin and New York, 1966. MR 36 \#5145.

6. D. Lazard, Épimorphismes plats d'anneaux, C. R. Acad. Sci. Paris Sér. A-B 266 (1968), A314-A316. MR 39 \#197c.

7. M. Raynaud, Anneaux locaux henséliens, Lecture Notes in Math., vol. 169, Springer-Verlag, Berlin and New York, 1970. MR $43 \# 3252$.

DEPARTMENT OF MATHEMATICS, UNIVERSITY OF ILLINOIS, URBANA, ILLINOIS 61801

Current address: Matematisk Institut, Københavns Universitet, Universitetsparken 5, DK2100 København ฤ, Danmark 